in diesem Zusammenhang als die bestmögliche deutsche Entsprechung des Begriffes der »conflictscape $\mathrm{zu}$ verstehen, der sich direkt aus Appadurais Konzept ableitet (Pospisil, 2020). »The suffix -scape allows us to point to the fluid, irregular shapes of these landscapes. (Appadurai, 1996: 33)

Fluid, irregulär und fragmentarisch, ineinander verwoben und doch in ihrem eigenen, spezifischen Charakter einzigartig, sind Konfliktlandschaften ein konzeptioneller Ansatz, der grundsätzlich jedem Kontext zugänglich ist. Er erweitert Appadurais ethnografisch-soziologische Sichtweise um das Element des eigentümlich institutionalisierten Feldes der Politik und der politischen Auseinandersetzung. Keinesfalls beschränkt sich der Begriff auf Gewaltkonflikte. Vielmehr schließt er, wie es Mary Kaldor in einer Diskussion um den Begriffsgehalt treffend bemerkt hat, Friedenslandschaften unabdingbar mit ein. Wenn sich das vorliegende Buch also der Konfliktlandschaften des Südsudan annimmt, geht es also keineswegs nur um Krieg und Gewalt. Es geht um eine Annäherung an einen vielschichtigen Kontext, in den unterschiedliche soziale Konfigurationen zusammengeworfen sind, ähnlich den vielschichtigen Bildern einer Landschaft und den diversen Formationen, die sie im Austausch mit Klima, Jahreszeiten, Wetter und humanen wie nichthumanen Einwirkungen annimmt.

Die Möglichkeiten, Konfliktlandschaften zu beschreiben, sind endlos. Von einer zeitlich, territorial, oder hinsichtlich der Dimension abgegrenzten Darstellung nehme ich Abstand. $\mathrm{Zu}$ viel der Wechselwirkungen würde angesichts dieser scheinbaren Ordnung verloren gehen. Die gewählte Vorgangsweise fokussiert daher auf den Charakter, den Inhalt, die besondere Gestalt dieser Konfliktlandschaften, jene Aspekte, die sie in Dynamik bringen und halten: Logiken und Felder.

\title{
Die Methodologie des Fragments
}

Eine umfassende analytische Beschreibung solcher Konstellationen ist ein hoffnungsloses Unterfangen. Es würde auf der Notwendigkeit von Typologisierungen und Kategorisierungen beruhen, welche wiederum ein konsistentes Ganzes als Referenzpunkt benötigen, um greifbar zu bleiben. Es ist daher unvermeidlich, eine solche Herangehensweise zu verwerfen. Die Formulierung einer Alternative ist jedoch um einiges schwieriger. »How does one write a book about the inability to understand without occasionally committing the same misdeed?« (Najslova, 2021: 11) 
Wie also ein Buch gestalten, das sich mit dem Südsudan und seinen Konfliktlandschaften auseinandersetzen soll? Vollkommen gerecht kann eine solche Darstellung der Realität nie werden. Dennoch gibt es bessere und schlechtere, einfühlsamere und weniger einfühlsame Möglichkeiten, sich dieser Aufgabe zu nähern. Schon eine oberflächliche Überlegung macht klar, dass weder eine stringente und wie immer komplex verschachtelte Analyse noch eine chronologisch angelegte historische Abhandlung gangbare Optionen der Darlegung bereitstellen.

Die Metapher der Landschaft hilft bei der Erarbeitung von Alternativen. Methodisch ist eine vollkommene Darstellung von Landschaften unmöglich. Karten geben uns einen verkleinerten und vereinfachten Ausschnitt, sie können verschiedene Formationen in ihrer Geworfenheit abbilden, aber nur in ihrer oberflächlichen, auf den ersten Blick sichtbaren Dimension. Es benötigt eine Verlagerung des Blicks von der umfassenden Einhegung hin zum Einlassen auf relatives Verstehen. David Chandler hat diese Veränderung der Perspektive mit dem Begriff des »Mapping « umschrieben, einem Ansatz, den er als alternativen Ansatz von Governance charakterisiert, und der die moderne Steuerungstechnik grundsätzlich infrage stellt. Mapping verschiebt den Fokus »from the subject of power (the ideas and understanding of governing agencies) to the importance of the object of governance itself « und akzeptiert so die Abkehr von liberalen Grundpfeilern wie Linearität und Universalität (Chandler, 2018: 21).

Ein solches Mapping erscheint als ein tragfähiger Ansatz für das vorliegende Unterfangen. Er kann auch auf einzelne Beispiele verweisen. Obwohl ich der Anthropologie und ihrer untilgbar kolonialen Herkunft kritisch gegenüberstehe, sind es oft gerade Ethnografien, die zu den methodisch einprägsamsten Werken gehören. Sie weisen in der Darstellung mitunter Wege, die klassische Sozialwissenschaft trocken und angestaubt zurücklassen. Für den vorliegenden Band waren es insbesondere zwei Ethnografien, die Methodik und Erzählweise prägen. Zunächst Anna Lowenhaupt Tsings bahnbrechende Studie über »The Mushroom at the End of the World«, eine kraftvolle Geschichte, die sich entschlossen gängigen wissenschaftlichen Darstellungsklischees verschließt. Tsing begibt sich auf die Suche nach Brüchen, Widersprüchen, Non-Linearität. Sie unternimmt ein Nachzeichnen von in sich widersprüchlichen Realitäten anstatt einer glatten und bestechenden Strukturanalyse. Nur so gelingt es ihr, den Weg des Matsutake, dieses seltsamen Pilzes, der sich von abgeschiedenen Regionen wie aufgegebenen Nutzwäldern in 
Oregon nach Japan aufmacht, um dort als Delikatesse Top-Preise zu erzielen, verständnisvoll darzustellen.

»In the intellectual woodlands I have been trying to encourage, adventures lead to more adventures, and treasures lead to further treasures. When gathering mushrooms, one is not enough, finding the first encourages me to find more." (Tsing, 2015: 287-288) Das Problem von Tsing ist eben nicht die Einebnung analytischer Pfade auf eine einheitliche Geschichte. Ihr zentrales Argument ist das der Pluralität von Realitäten und Erzählwegen, nicht verstanden als postmodern argumentierte Stringenz, sondern als perspektivische Diversität.

Das zweite Buch, das die vorliegende Arbeit methodologisch geprägt hat, ist Lucia Najslovas (2021) Annäherung an das beständige türkisch-EUeuropäische Missverständnis, »Turkey and the European Union: The Politics of Belonging«. Angesiedelt zwischen Ethnografie, politischer Analyse und hintergründigem Journalismus arbeitet Najslova dieses schwierige, widersprüchliche Verhältnis in nur lose zusammenhängenden Vignetten auf. Die Fragmente des Missverstehens, die sie schildert, erheben keinen Anspruch auf Vollständigkeit und Repräsentanz. Vielleicht eröffnen sie aber gerade deswegen einen innovativen Blick auf eine Konstellation, zu der eigentlich alles gesagt $\mathrm{zu}$ sein schien. Fragmente werden von Najslova konsequent zur Methodik erhoben, und in der Tat, »fragmented stories may well be the best thing to share, once the grand narratives have started falling apart (Najslova, 2021: 11).

Fragmente als Methodologie übersetzen sich im gegenständlichen Fall in eine fragmentarische Methodik. Im Gegensatz zu Tsing und Najslova kann ich allerdings nicht mit einer geplanten Ethnografie aufwarten. Ganz im Gegenteil. Dem vorliegenden Buch liegt keine spezifische Forschung zugrunde. Es ist ein Nebenprodukt aus unterschiedlichsten Vorhaben, die mich in den vergangenen zwölf Jahren immer wieder in den Südsudan geführt und mit dem Land beschäftigt haben. Die methodische Vorgangsweise war zumeist klassisch qualitativ. Interviews, Gespräche, Dokumentenanalysen. Über die Jahre ergänzen sich diese traditionellen methodischen Elemente mit Freundschaften, Begegnungen, Eindrücken, vertieften Auseinandersetzungen mit Kontexten, Elemente, die im sozialwissenschaftlichen Duktus für gewöhnlich als teilnehmende Beobachtung charakterisiert werden. Ein Feldtagebuch existiert nicht. Die Reflexion der Eindrücke erfolgt aus der Erinnerung, nur wörtliche Zitate sind Wiedergaben aus Notizen, festgehalten kurz nach den entsprechenden Konversationen. 
Die Freude der Arbeit an einer Monografie ist die relative methodische Freiheit, die sie bereithält. Dieses Buch nimmt sich diese Freiheit. Es ist nach strikten Kriterien als nur peripher wissenschaftlich zu charakterisieren und hätte in einem Journal-Peer-Review-Prozess vermutlich mit relevanten Schwierigkeiten $\mathrm{zu}$ rechnen. Gerade deswegen ist es notwendig, die drei Zielsetzungen, die das Unterfangen verfolgt, klar darzulegen: Interessierten unterschiedlicher Provenienz einen Einblick in mögliche Wahrnehmungen des südsudanesischen Kontexts zu bieten, die Diskussion um nachmoderne Ansätze in der Friedens- und Konfliktforschung anhand eines konkreten Kontexts voranzutreiben, und, letztendlich, das egoistische Motiv, mir selbst die Gelegenheit $\mathrm{zu}$ einer Reflexion meiner erlebten Forschung zu bieten. Auch wenn eine vollumfängliche Zielerreichung wohl nicht möglich ist, war dies doch die Intention, die hinter dieser Arbeit steht.

\section{Fahrplan}

Gemäß der dargestellten Methodologie erschließt sich der Inhalt dieser Monografie nicht chronologisch. Vielmehr bilden die folgenden elf Abschnitte in sich abgeschlossene Erzählungen, die in den Kontext Südsudan aus unterschiedlicher Perspektive eindringen. Diese Vorgangsweise erhebt nicht den Anspruch, eine Erzählung zu generieren, an deren Ende ein systematisches Verständnis steht.

Dennoch versuchen sich die folgenden drei Kapitel in einer gerafften Version einer weitgehend einheitlich erzählten Geschichte des Südsudan. Es ist eine im historischen Institutionalismus verankerte Diskussion der Entwicklung von Fragmenten einer nationalstaatlichen Ordnung, die den heutigen südsudanesischen Staat kennzeichnet. Diese Erzählung verfolgt einen doppelten Zweck: einerseits ist dieser Staat manifest, wenngleich in spezifischen und oftmals unerwarteten Formen und Praktiken. Diese Formen und Praktiken zu kennen und geschichtlich zurückzuverfolgen ist ein wichtiger Baustein zur Annäherung an südsudanesische Realitäten. Andererseits liefert die Darstellung ein Grundgerüst, das die Einordnung der folgenden Vignetten erleichtert, insbesondere für Leser:innen, die mit der Materie weniger vertraut sind.

Die folgenden fünf Kapitel widmen sich spezifischen Konfliktlandschaften, wobei deren Darstellung weder als vollständig noch als abgeschlossen $\mathrm{zu}$ lesen ist. Die erste derartige Konfliktlandschaft beschäftigt sich mit über 